\title{
Top-quark physics at the first CLIC stage
}

\author{
Aleksander Filip Żarnecki ${ }^{* \dagger}$ on behalf of the CLICdp Collaboration \\ Faculty of Physics, University of Warsaw \\ E-mail: Filip.Zarnecki@fuw.edu.pl
}

\begin{abstract}
The Compact Linear Collider (CLIC) is a mature option for a future electron-positron collider operating at centre-of-mass energies of up to $3 \mathrm{TeV}$. CLIC will be built and operated in a staged approach with three centre-of-mass energies currently assumed to be $380 \mathrm{GeV}, 1.5 \mathrm{TeV}$ and $3 \mathrm{TeV}$. The energy of the initial stage was chosen to optimize the physics potential in terms of Higgsboson and top-quark measurements. This contribution discusses the prospects for precision measurements of top-quark properties at the first stage of CLIC, based on detailed simulation studies, taking into account luminosity spectra and beam induced backgrounds, full detector simulation based on GEANT4, final state reconstruction based on particle flow approach with PANDORAPFA, jet clustering with the VLC algorithm as implemented in the FASTJET package, and flavour tagging with LCFIPLUS.

Based on a dedicated centre-of-mass energy scan around the top-quark pair production threshold, the top-quark mass can be determined with a precision of about $50 \mathrm{MeV}$ in a theoretically welldefined manner. This scan is also sensitive to the top-quark width and Yukawa coupling. Other approaches to extract the top-quark mass at the first stage of CLIC make use of ISR photons or the direct reconstruction of the top quarks. Precise measurements of the differential top-quark pair production cross sections at $380 \mathrm{GeV}$, for different electron beam polarisations, allow the study of top-quark couplings to electroweak gauge bosons. Expected limits on new physics contributions described in terms of Effective Field Theory (EFT) operator coefficients are presented, showing sensitivity of the first CLIC stage to mass scales beyond $10 \mathrm{TeV}$. The large number of top-quark pairs produced also allows competitive searches for Flavour Changing Neutral Current (FCNC) top-quark decays with charm quarks in the final state. Exclusion limits expected for $500 \mathrm{fb}^{-1}$ collected at the first stage of CLIC are presented for $\mathrm{t} \rightarrow \mathrm{cH}, \mathrm{t} \rightarrow \mathrm{c} \gamma$ and $\mathrm{t} \rightarrow \mathrm{cE}$ channels, reaching down to $4.7 \cdot 10^{-5}$ for $\mathrm{BR}(\mathrm{t} \rightarrow \mathrm{c} \gamma)$.
\end{abstract}

The 39th International Conference on High Energy Physics (ICHEP2018)

4-11 July, 2018

Seoul, Korea

\footnotetext{
${ }^{*}$ Speaker.

${ }^{\dagger}$ Supported by the National Science Centre, Poland, project nr 2017/25/B/ST2/00496.
} 


\section{Introduction}

Processes involving top quarks provide us unique opportunities to test the Standard Model (SM) predictions and look for possible signatures of new physics beyond the SM (BSM). Thorough studies of the top-quark physics potential of CLIC, based on detector-level simulations, have been recently presented by the CLIC Detector and Physics collaboration [1]. Summarized in this contribution are the main top-quark physics results expected after the initial stage of CLIC operation at $380 \mathrm{GeV}$. Prospects for top-quark physics at high-energy CLIC stages are covered in [2].

\section{Top-quark mass measurements}

The most precise determination of the top-quark mass, both in terms of the statistical and of the systematic uncertainties, is possible with a dedicated scan of the top pair production threshold. The shape of the pair-production cross section around the threshold is very sensitive not only to the top-quark mass, but also to its width and Yukawa coupling. Although the dependence is smeared by ISR effects and the beam luminosity spectrum, $100 \mathrm{fb}^{-1}$ collected around the threshold with the dedicated luminosity spectrum is sufficient to reduce the statistical mass uncertainty (assuming fixed width and Yukawa coupling) to $19 \mathrm{MeV}$, see figure 1 (left). The top-quark mass is extracted in a well-defined theoretical scheme, significantly reducing the systematic uncertainties. Combined theoretical uncertainties, including parametric uncertainty from $\alpha_{s}$ and from QCD scale variations, are estimated to be between $30 \mathrm{MeV}$ and $50 \mathrm{MeV}$. Experimental systematic uncertainties, including beam energy and the luminosity spectrum, selection efficiencies and residual background levels, are estimated to be at the similar level, between $25 \mathrm{MeV}$ and $50 \mathrm{MeV}$. A total systematic uncertainty on the top-quark mass determination from the threshold scan of around $50 \mathrm{MeV}$ is therefore feasible.

The top-quark mass can also be extracted when running above the threshold, at $380 \mathrm{GeV}$, from the cross section of radiative events, $\mathrm{e}^{+} \mathrm{e}^{-} \rightarrow \mathrm{t} \overline{\mathrm{t}} \gamma$. The expected distribution of the reconstructed top-pair invariant mass is presented in figure 1 (right). With the assumed integrated luminosity of $500 \mathrm{fb}^{-1}$ a statistical precision of about $100 \mathrm{MeV}$ can be obtained and the total uncertainty is estimated to be about $150 \mathrm{MeV}$.

The direct top-quark mass determination from the reconstructed decay products is accessible at all CLIC energy stages. Statistical precision of around $40 \mathrm{MeV}$ is expected at the first CLIC stage for combined hadronic and semi-leptonic top-quark pair production samples. To match the statistical accuracy the jet energy scale should be controlled at the level of $0.025 \%$. Jet energy scale effects can be significantly reduced by relating the top-quark mass to the $\mathrm{W}$ boson mass. The expected statistical uncertainty on the reconstructed mass ratio corresponds to a top-quark mass uncertainty of about $60 \mathrm{MeV}$.

\section{Properties of top-quark pair production}

Top-quark couplings to the photon and the Z-boson can already be precisely determined at

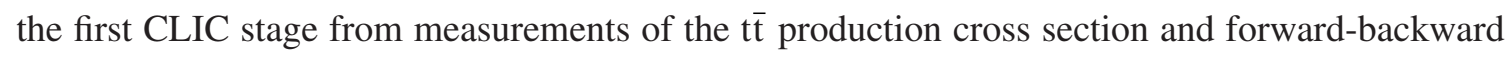
asymmetry with different electron beam polarisations. The pair production cross section can be measured for both polarisations with statistical accuracy below $1 \%$ (assuming $250 \mathrm{fb}^{-1}$ per polarisation) while the forward-backward asymmetry is extracted from the measured top-quark angular 
distribution for semi-leptonic events with a statistical uncertainty of the order of 4-5\%, see figure 2 (left). Top-quark measurements can be used to constrain the possible BSM effects induced by heavy new physics described in terms of Effective Field Theory (EFT) operators. Expected limits on the Wilson coefficients for seven EFT operators contributing to the top-quark pair production are presented in figure 2(right). Even at the first CLIC stage mass scales in the $10 \mathrm{TeV}$ range can be probed. For four operators, most of the sensitivity is provided by the initial $380 \mathrm{GeV}$ stage.

\section{Flavour-changing neutral current top-quark decays}

FCNC top-quark decays are very strongly suppressed in the SM, with expected branching ratios of the order of $10^{-14}$ to $10^{-12}$. On the other hand, significant enhancement is expected in many BSM scenarios, reaching up to $10^{-2}$ for BR $(\mathrm{t} \rightarrow \mathrm{cH})$ and $10^{-5}$ for BR $(\mathrm{t} \rightarrow \mathrm{c} \gamma)$. Searches for FCNC top decays at CLIC, for channels involving charm quark, can also be competitive with the expected HL-LHC reach for these channels of around $2 \cdot 10^{-4}$ [3] and $7 \cdot 10^{-5}$ [4], respectively.

Three FCNC decay channels have been studied for the first stage of CLIC. All channels profit from the precise final state reconstruction and high flavour tagging efficiency expected for the CLIC detector. For $\mathrm{t} \rightarrow \mathrm{c} \gamma$ decay we expect a high energy isolated photon and a c-quark jet in the final state, as well as a b-quark jet and a pair of light jets from the hadronic decay of the second ("spectator") top. The same hadronic final state topology is expected for the $t \rightarrow c \notin$ channel, but with no photon, and large missing energy and momentum from the escaping massive scalar particle. Finally, candidate events for $\mathrm{t} \rightarrow \mathrm{cH}$ decay (followed by Higgs boson decay $\mathrm{H} \rightarrow \mathrm{b} \overline{\mathrm{b}}$ ) are selected by looking for a c-quark jet and three b-quark jets, with invariant mass of two b-quark jets consistent with the $\mathrm{H}$ mass.

The same analysis procedure is applied for all channels. Event pre-selection and classification is based on global event properties, jet clustering results, flavour tagging as well as lepton and photon identification and isolation requirements. A kinematic fit for signal and background hypothesis is then performed for events matching the required signal final state topology. Expected limits are then calculated using $\mathrm{CL}_{\mathrm{s}}$ approach, based on the response distribution of the Boosted Decision Tree (BDT) classifier trained to discriminate between signal and background events. An example distribution of the BDT classifier response for events with FCNC top-quark decay $\mathrm{t} \rightarrow \mathrm{c} \gamma$ and SM background samples is presented in figure 3 (left). Expected 95\% C.L. limits for $500 \mathrm{fb}^{-1}$ collected at $380 \mathrm{GeV}$ CLIC are: $\mathrm{BR}(\mathrm{t} \rightarrow \mathrm{c} \gamma)<4.7 \cdot 10^{-5}, \mathrm{BR}(\mathrm{t} \rightarrow \mathrm{cH}) \times \mathrm{BR}(\mathrm{H} \rightarrow \mathrm{b} \overline{\mathrm{b}})<1.2 \cdot 10^{-4}$ and $\mathrm{BR}(\mathrm{t} \rightarrow \mathrm{cE})<1.2-4.1 \cdot 10^{-4}$, see figure 3 (right).

\section{References}

[1] H. Abramowicz et al. [CLICdp Collaboration], CLICdp-Pub-2018-003, arXiv:1807.02441 [hep-ex].

[2] U. Schnoor, Top-quark physics at high-energy CLIC operation, these proceedings.

[3] ATLAS Collaboration, Expected sensitivity of ATLAS to FCNC top quark decays $t \rightarrow Z u$ and $t \rightarrow H q$ at the High Luminosity LHC, ATL-PHYS-PUB-2016-019.

[4] CMS Collaboration, Technical Design Report of the endcap calorimeter for the Phase-2 upgrade of the CMS experiment, in view of the HL-LHC run, CERN-LHCC-2017-023, CMS-TDR-019. 

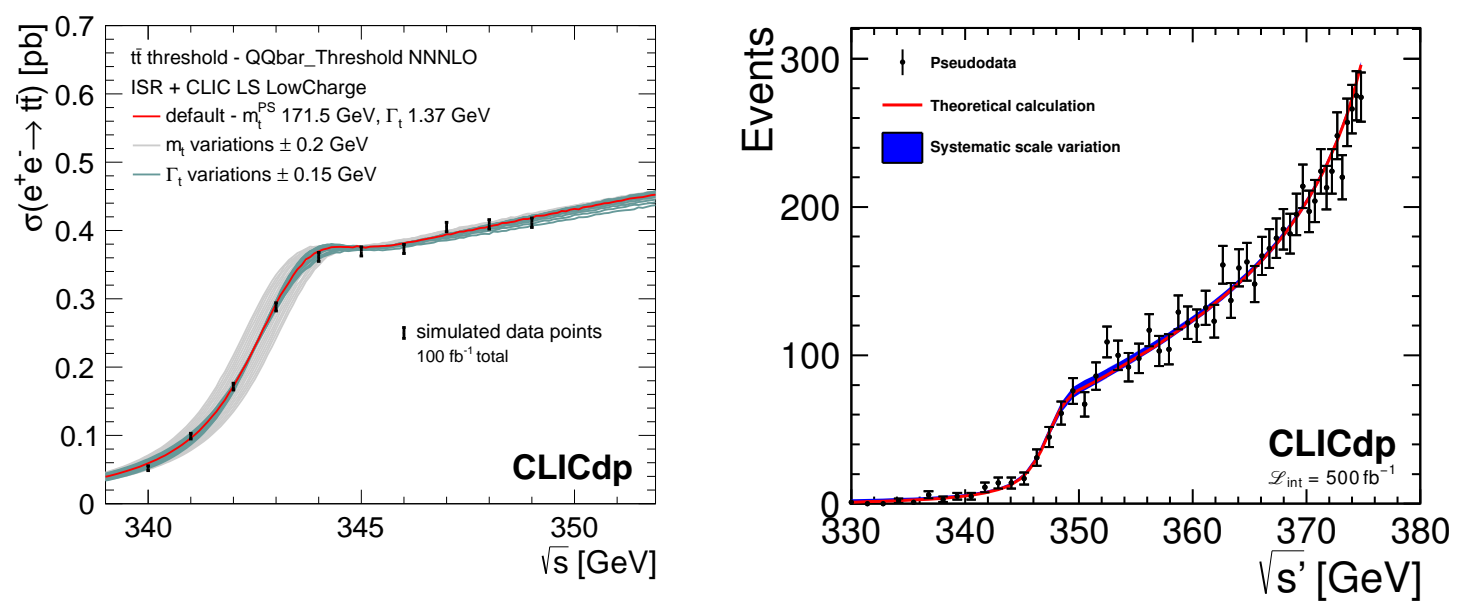

Figure 1: Illustration of measurements allowing a top-quark mass determination at the first stage of CLIC: from the dedicated threshold scan (left) and from radiative event reconstruction at $380 \mathrm{GeV}$ (right). Figures taken from [1].
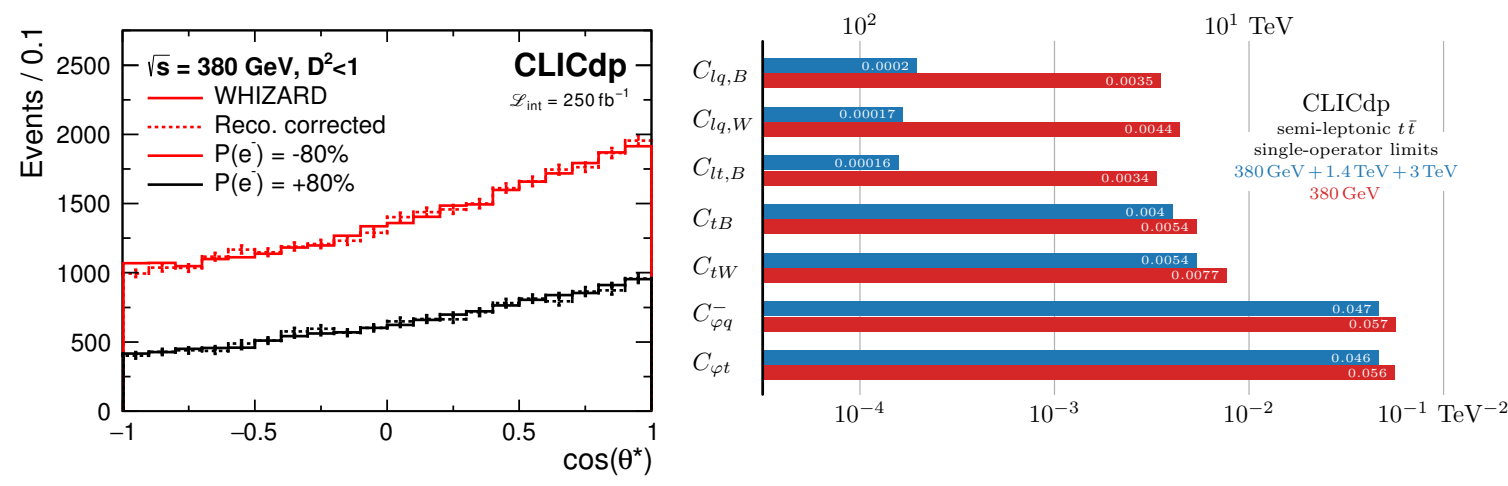

Figure 2: Top-quark polar angle distributions for operation at $\sqrt{s}=380 \mathrm{GeV}$ after the application of a tight quality cut (left) and the expected limits on the Wilson coefficients from the global EFT analysis of top-quark pair production at the $380 \mathrm{GeV}$ (right). Figures taken from [1].
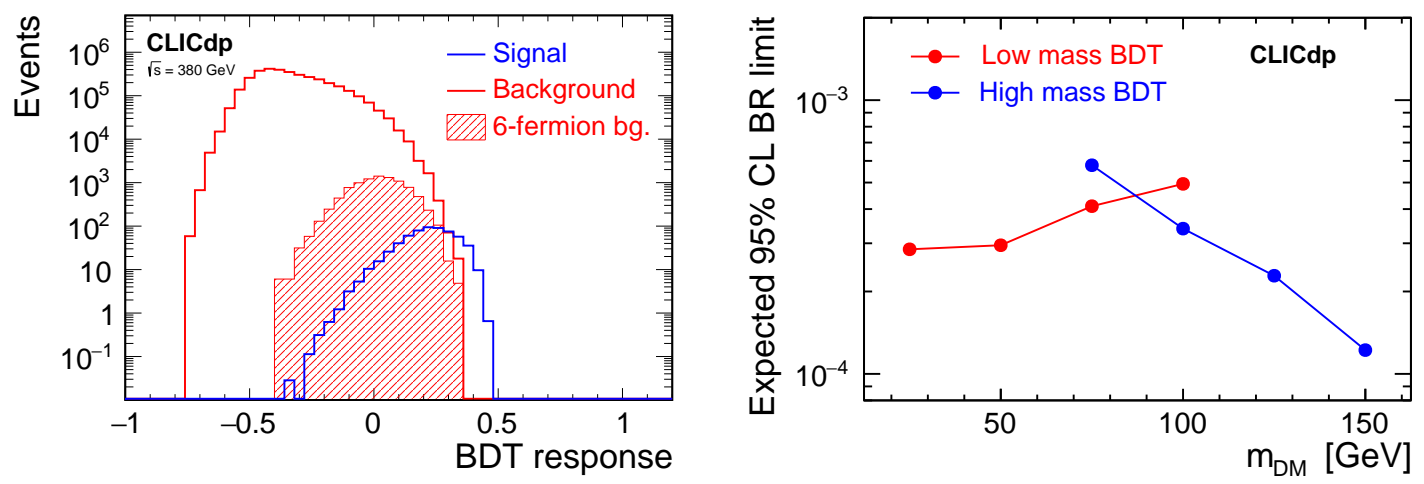

Figure 3: Distribution of the BDT classifier response for events with FCNC top-quark decay $t \rightarrow c \gamma$ and SM background samples (left) and the expected 95\% C.L. limits on the top quark FCNC decay $\mathrm{t} \rightarrow \mathrm{cE}$ for $500 \mathrm{fb}^{-1}$ collected at $380 \mathrm{GeV}$ CLIC (right). Figures taken from [1]. 